\title{
Simulações espacialmente explícitas dos estoques de carbono orgânico de um LATOSSOLO BRUNO por meio da integração do modelo century com GIS
}

\author{
Spatially explicit simulations of soil carbon stocks in Humic Hapludox, using Century model and GIS
}

\author{
Fabíola Lopes $^{{ }^{*}}$ João Mielniczuk $^{\mathrm{I}}$ Elisandra Oliveira Bortolon ${ }^{\mathrm{II}}$ \\ Carlos Gustavo Tornquist ${ }^{\mathrm{I}}$ Elvio Giasson ${ }^{\mathrm{I}}$
}

\section{RESUMO}

Os modelos de simulação, uma vez calibrados localmente, são ferramentas úteis para a avaliação dos impactos da agricultura sobre a dinâmica do carbono orgânico do solo (COS) e planejamento de sistemas agrícolas sustentáveis em escala regional. O presente trabalho teve como objetivo estudar a dinâmica do COS em um LATOSSOLO BRUNO Aluminoférrico típico muito argiloso, localizado em uma região de clima subtropical, por meio de simulações com o modelo Century versão 4.0, apoiado em levantamentos históricos e técnicas de geoprocessamento e sensoriamento remoto. Verificou-se que, para o período atual, a mudança de uso do solo, representada pela conversão da vegetação nativa em agricultura, causou uma redução no conteúdo de carbono ao longo dos anos, sendo que as lavouras com maior tempo de exploração e submetidas ao preparo convencional apresentaram os menores estoques. Por outro lado, nas simulações futuras (ano 2058), verifica-se que as lavouras, quando sob manejos conservacionistas, podem recuperar, e até superar, os estoques de COS encontrados no solo sob vegetação nativa de campo.

Palavras-chave: manejo e uso, campo nativo, carbono no solo, modelo Century.

\section{ABSTRACT}

Simulation models are useful tools for assessment of the impacts of agriculture on nutrient and soil organic C (SOC) dynamics. Results of simulation studies can be applied to develop sustainable agricultural systems. This study simulated SOC in clayey Humic Hapludox with Century model (version 4.0) in a subtropical climate, with support from historical soil and land-use surveys; GIS and remote sensing techniques. Major reductions in SOC stocks were observed after land under native vegetation was converted to agricultural use, especially under annual crops managed under conventional tillage. Simulations of these soils under current management to the year 2058 showed that soils under conservation systems (especially no tillage) can recover and in some cases even exceed the original SOC stocks under native vegetation.

Key words: land use change, native vegetation, soil C, Century Model

\section{INTRODUÇÃO}

Estudos da evolução espaço-temporal, por meio da espacialização do uso do solo, mostraramse fundamentais em trabalhos de avaliação da dinâmica dos estoques de COS e, consequentemente, da qualidade do solo (TORNQUIST et al., 2009). Trabalhos associando o modelo Century (PARTON et al., 1987) e o Sistema de Informação Geográfica (SIG), visando à espacialização dos estoques de COS, já foram desenvolvidos e testados nas condições do Rio Grande do Sul (RS) (LOPES et al., 2008; TORNQUIST et al., 2009; BORTOLON et al., 2011). LOPES et al. (2008) utilizou a modelagem espacialmente explícita para avaliar a dinâmica espaço-temporal do COS na escala de pequena bacia hidrográfica rural, localizada na encosta superior do nordeste do RS, e caracterizada por possuir clima com temperatura média máxima anual de $24^{\circ} \mathrm{C}$ e média mínima anual de $12^{\circ} \mathrm{C}$, precipitação média anual de $1.980 \mathrm{~mm}$ e solos com $38 \%$ de teor médio de argila. TORNQUIST et al. (2009) e BORTOLON et al. (2011) aplicaram o modelo Century em condições da região do Planalto Rio-grandense, em solos argilosos, com teor máximo de argila de $70,7 \%$ e sob clima de temperatura média anual em torno de

IDepartamento de Solos, Faculdade de Agronomia, Universidade Federal do Rio Grande do Sul (UFRGS), 90040-060, Porto Alegre, RS, Brasil. E-mail: fabilopes@gmail.com.*Autor para correspondência.

${ }^{\text {II } C e n t r o ~ N a c i o n a l ~ d e ~ P e s c a, ~ A q u i c u l t u r a ~ e ~ S i s t e m a s ~ A g r i ́ c o l a s, ~ E m p r e s a ~ B r a s i l e i r a ~ d e ~ P e s q u i s a ~ A g r o p e c u a ́ r i a ~(E M B R A P A), ~ P a l m a s, ~ T O, ~}$ Brasil. 
$19^{\circ} \mathrm{C}$ e precipitação média de $1.720 \mathrm{~mm}$. Em todos os trabalhos citados, o Century, associado à SIG, mostrou-se uma ferramenta adequada para auxiliar no estudo das alterações do COS, no tempo e espaço.

Entre as diversas condições de clima e solo existentes no país, a situação de solos muito argilosos, ricos em óxidos de ferro e alumínio, associada a clima frio e úmido, os quais proporcionam uma maior proteção da matéria orgânica do solo (MOS) e da decomposição microbiana, ainda não foi testada no modelo Century. Diante disso, neste trabalho, propôs-se estimar regionalmente os estoques de COS e sua dinâmica em um solo localizado na região de Vacaria, RS, por possuir essas características de clima e solo diferenciados.

\section{MATERIAL E MÉTODOS}

Caracterização geral da área de estudo e amostragem: a área possui $126 \mathrm{~km}^{2}$ e está situada ao norte do Município de Vacaria, RS (28³0’45,40”S, $51^{\circ} 01$ '13,58’W). A vegetação predominante na região de estudo foi originalmente composta por capões e restingas nos vales e várzeas; densa mata subtropical nas encostas dos rios e, principalmente, por campos limpos, com a presença do pinheiro araucária (Araucaria angustifolia). Os dados climáticos médios correspondem à precipitação média anual de $1.500 \mathrm{~mm}$, temperatura média mensal máxima de $22^{\circ} \mathrm{C}$ e a média mensal mínima de $10,2^{\circ} \mathrm{C}$, sendo que o mês mais frio apresenta média de $5,7^{\circ} \mathrm{C}\left(8^{\circ}\right.$ DISME/INMET).

Como a região de interesse encontra-se numa porção homogênea da paisagem, sem variações abruptas do terreno, e os mapas de levantamento de solo em escalas maiores são ainda escassos no país, neste trabalho, optou-se pela utilização do levantamento de reconhecimento de solos do RS na escala de 1:750.000 (BRASIL, 1973). Por meio deste, identificou-se o LATOSSOLO BRUNO Aluminoférrico típico, pertencente à unidade de mapeamento Vacaria, como o predominante na região. As variáveis de solo usadas no modelo foram obtidas a partir de amostragem de campo e resultaram nos valores médios de granulometria, obtidos pelo método da pipeta (EMBRAPA, 1997), de 76,98\% de argila; $19,40 \%$ de silte; e $3,62 \%$ de areia. Segundo POTTER (1977), a mineralogia dominante é composta por caulinita e óxidos de ferro.

Os estoques de COS foram calculados pela equação Estoque $=\mathrm{C} \times \rho \times$ prof. $\times 10$, em que: Estoque $=$ estoque de $\operatorname{COS}\left(\mathrm{Mg} \mathrm{ha}^{-1}\right) ; \mathrm{C}=$ concentração de carbono na amostra $\left(\mathrm{kg} \mathrm{Mg}^{-1}\right) ; \rho=$ densidade do solo $\left(\mathrm{Mg} \mathrm{m}^{-3}\right)$; prof. $=$ espessura da camada $(\mathrm{m})$.
Uso do solo: segundo informações de agricultores e dos agrônomos atuantes na região, juntamente com os dados dos anuários agrícolas do Instituto Brasileiro de Geografia e Estatística IBGE, em meados de 1950, os solos da região eram praticamente todos cobertos por campo nativo, tendo como principal atividade a pecuária extensiva. Verificou-se que no ano de 1964: 9,5\% da área já estavam ocupadas com agricultura; em 1976: 17\% da área; em 1987: 23,4\%; em 1996: 34,5\%; chegando, em 2005, a aproximadamente $40 \%$ da área explorada com agricultura (LOPES et al., 2010).

Elaboração das unidades de simulação (US): a agricultura na região de Vacaria iniciou mais tardiamente, em meados da década de 1950, e, devido a essa característica, tornou-se possível a reconstituição da ocupação espaço-temporal do solo, realizada exclusivamente por meio de produtos do sensoriamento remoto. Assim, de acordo com LOPES et al. (2010), foi realizada a abordagem detalhada da identificação espacializada das mudanças de uso agrícola dos solos da região de estudo, separando-se as áreas de lavouras desde 1964, 1976, 1987, 1996 e 2005. Os mapas especializados detalhados, com todos os períodos estudados, estão em LOPES et al. (2010).

O relevo da área de estudo, representado pelas diferentes posições na paisagem, foi espacializado, levando em consideração as informações contidas nos mapas de altitude e declividade. A declividade foi obtida por meio do modelo numérico do terreno (MNT). Com o auxílio das informações contidas nesses dois mapas, construiu-se um mapa final, separando as áreas de topo (com declividade entre 0 e $<8 \%$ ), de encosta (com declividade $>8 \%$ ) e as áreas deposicionais (com declividade próxima a zero, que não é topo).

Para estudar o comportamento dos estoques de COS ao longo do tempo, dividiu-se a área de estudo em áreas homogêneas, denominadas unidades de simulação (US). Nessa divisão, consideraram-se o tempo de agricultura e os elementos da paisagem. Assim, cada US foi espacialmente representada em um mapa temático e, posteriormente, foi realizada a simulação com cenários de manejo bem definidos pelo modelo Century. O mapa final com as diferentes US foi obtido por meio de cruzamentos, no ArcGIS (ESRI, 2003), do mapa das diferentes posições na paisagem, pelos períodos de conversão em agricultura, originando as seguintes US: Topo com lavoura desde 1964; Topo com lavoura desde 1976; Topo com lavoura desde 1987; Topo com lavoura desde 1996; Topo com lavoura desde 2005; Encosta 
com lavoura desde 1964; Encosta com lavoura desde 1976; Encosta com lavoura desde 1987; Encosta com lavoura desde 1996; e Encosta com lavoura desde 2005 (LOPES et al., 2010).

Modelagem - Execução de equilíbrio sob campo nativo: na etapa inicial, chamada de "execução de equilíbrio", o Century foi executado por um período de 6000 anos na condição de campo nativo, verificando-se, ao final desse período, se os estoques de COS estimados pelo modelo (variável SOMSC) correspondiam aos estoques reais médios, presentes na camada de 0 a $20 \mathrm{~cm}$ do solo sob vegetação nativa. A correspondência entre o valor de CO observado no solo sob campo nativo e o valor estimado pelo Century foi obtida por meio de ajustes nos parâmetros locais, de cultura e parâmetros fixos, conforme descrito abaixo.

Parâmetro local: EPNFS(2) - Entrada de N pela fixação não-simbiótica - original: 0,028 modificado: 0,019. Parâmetro de cultura: PRDX (g $\mathrm{C} \mathrm{m}^{-2}$ mês $\left.^{-1}\right)$ - Potencial máximo mensal de produção de $\mathrm{C}$ pela parte aérea. No caso deste trabalho, para o ecossistema "campos subtropicais" - original: * modificado: 488. Parâmetros fixos: VARAT2 $(1,1)$ Relacionado às relações $\mathrm{C} / \mathrm{N}$ do material que entra no compartimento lento - original: 18 - modificado: 20. VARAT3(1,1) - Relacionados às relações $\mathrm{C} / \mathrm{N}$ do material que entra no compartimento passivo original: 8 - modificado: 10. VLOSSG - Perda de N por volatilização - original: 0,010 - modificado: 0,013 . PS1S3(2) - Fluxo de C do compartimento ativo para o passivo - original: 0,032 - modificado: 0,05. PS2S3(2) - Fluxo de C do compartimento passivo para o lento - original: 0,009 - modificado: 0,012.

Os parâmetros PS1S3(2) e PS2S3(2) foram alterados visando a adequar a porcentagem de $\mathrm{CO}$ que é distribuída entre os compartimentos passivo e lento aos valores esperados para um solo muito argiloso, com presença de óxidos de alumínio e ferro, associado a um clima mais ameno, que proporcionam uma maior proteção da MOS à decomposição microbiana. Foi criado no arquivo de cultura (CROP.100) um campo nativo com as características do campo nativo encontrado na região de Vacaria.

Calibração e validação do modelo: após a simulação de equilíbrio, executou-se a calibração, visando a chegar ao estoque de COS referente à lavoura mais antiga disponível na região de interesse, representando a US Topo, com lavoura desde 1964. A acurácia das simulações foi avaliada com a estatística RMSE - Root Mean Square Error of model (SMITH et al.,1997), sendo utilizados dados de campo diferentes daqueles usados nas etapas iniciais de equilíbrio e calibração.
Execução do modelo no período agrícola: nesta etapa, procedeu-se aos ajustes adicionais dos parâmetros de cultura(crop.100)e de cultivo(cult.100), com base nos estoques das lavouras iniciadas em 1964. Assim: Parâmetros de cultura - PRDX (g C m-2 mês-1): Trigo - W1: original: 250 modificado: 170; Trigo - W2: original: 250 modificado: 220; Trigo W3: original: 300 modificado: 250; Soja - SYBN2: original: 300 modificado: 175; Soja - SYBN3: original: 300 modificado: 195; Soja - SYBN4: original: 450 modificado: 200; Aveia - OAT 2: original: 320 modificado: 380; Pousio - Weeds: original: 300 modificado: 150. Parâmetros de cultivo: CLTEFF(2) - Multiplicador que aumenta a decomposição do referido compartimento no mês de cultivo: original: 1,60 modificado: 3,20.

Além das alterações citadas acima, para adequar o modelo para condições de solos cultivados, utilizou-se um "efeito adicional de cultivo" (CULT A), por quatro meses seguidos após o revolvimento do solo no plantio convencional e por três meses no preparo reduzido, tendo-se prolongado, assim, o efeito do preparo sobre as taxas de decomposição da MOS (BORTOLON et al., 2009).

Para as simulações, foram fornecidas as seguintes variáveis de entrada: média mensal da temperatura (máx. e mín.) e da precipitação; granulometria das áreas de campo e lavoura; quantidade de COS dos compartimentos ativo, lento e passivo (obtida pela execução de equilíbrio); dados de campo nativo (criado no arquivo crop.100); e perdas de solo por erosão, onde se admitiram perdas de solo entre 1,5 a $6,0 \mathrm{Mg} \mathrm{ha}^{-1}$ (DEBARBA, 2002), aplicadas em dezembro de cada ano.

Na simulação das alterações no estoque de COS, a partir da condição de sistema estável (campo nativo), foram elaborados cenários de manejo que visaram à máxima semelhança com os manejos realizados pelos agricultores na área entre os anos de 1950 e 2008. Assim: de 1951 a 1970 - Preparo convencional (PC) com trigo com baixo rendimento e pousio; de 1971 a 1980 - PC com trigo/soja cultivares com médio potencial de rendimento; de 1981 a 1990 - Preparo reduzido (PR) com trigo/soja, cultivares com médio potencial de rendimento; de 1991 a 2058 - Sistema de Plantio Direto (PD), com trigo/soja aveia/soja aveia/milho - cultivares com alto potencial de rendimento.

As US partiram do estoque de $96,5 \mathrm{Mg}$ $\mathrm{ha}^{-1}$ de $\operatorname{COS}$ na camada de $0-20 \mathrm{~cm}$. Esse estoque foi obtido por meio da parametrização do Century, com a execução de equilíbrio, e foram aproximados dos valores de COS obtidos em análises de amostras 
dos solos sob vegetação nativa. A espacialização dos dados originados pelo Century foi realizada pela sua integração em um ambiente SIG, construindo assim um mapa de distribuição espacial de COS para toda a região de estudo no período entre 1950 a 2008.

\section{RESULTADOS E DISCUSSÃO}

Calibração do Century nas unidades de simulação: a calibração foi feita por meio das alterações dos parâmetros descritos nos itens 2.3 e 2.4 (Figura 1). O valor da RMSE obtido indica que a diferença entre os valores medidos e simulados foi pequena, visto que, quanto menor a raiz, menos intensas são as diferenças entre os valores estimados e observados (SMITH et al., 1997).

As adições médias de $\mathrm{C}$ pelas culturas estimadas pelo modelo variaram em função dos diferentes sistemas de culturas utilizados em cada período considerado, sendo que a menor adição anual de $\mathrm{C}$ ao solo foi de $2,24 \mathrm{Mg} \mathrm{ha}^{-1}$ no período de $\mathrm{PC}$, o que pode ser atribuído ao revolvimento e ao cultivo da sucessão trigo/pousio, o que limitou a adição de C. A maior adição total de $\mathrm{C}$ estimada ocorreu no $\mathrm{PD}$, com $5,17 \mathrm{Mg} \mathrm{ha}^{-1} \mathrm{ano}^{-1}$ com a sucessão de culturas trigo/soja aveia/soja e aveia/milho. Resultados semelhantes de adições foram obtidos em simulações com Century em outros solos do RS (TORNQUIST et al., 2009; BORTOLON et al., 2011).

Execução do modelo nas demais US de topo e encosta: neste estudo, as diferenças entre topo e encosta ocorrem unicamente pela variação das taxas de erosão adotadas em cada posição. Assim, essas diferentes posições tiveram um comportamento semelhante ao longo do tempo (Figura 2).

Observa-se uma grande queda inicial nos estoques de COS (Figura 2), o que é explicado pela presença de frações orgânicas com diferentes graus de estabilidade e/ou proteção à decomposição no solo. Sendo assim, logo após a alteração da vegetação nativa para o sistema agrícola, ocorre uma rápida decomposição da fração lábil da MO. As frações orgânicas sofrem também grande influência do PC, que provoca a ruptura dos agregados, expondo as frações que estavam protegidas no interior destes ao ataque microbiano. Após um determinado período de tempo, os compartimentos lábil e protegido estruturalmente tendem a atingir uma nova condição estável, porém bastante inferior à condição original, permanecendo no solo frações orgânicas mais resistentes à decomposição, como aquelas protegidas pela interação com a fração mineral do solo. Com isso, a atividade microbiana também é reduzida, aumentando a influência do processo erosivo nas perdas de $\mathrm{CO}$ do solo.

Nas lavouras iniciadas em 1976 (Figura 2a), observa-se uma queda nos estoques de COS, quando comparado com o estoque do campo nativo até o ano de 2008, havendo uma recuperação nos estoques apenas nas simulações futuras até 2058, quando ocorre um aumento de 5,1\% nos estoques de COS. Do ano de maior queda dos estoques (1991) até o presente

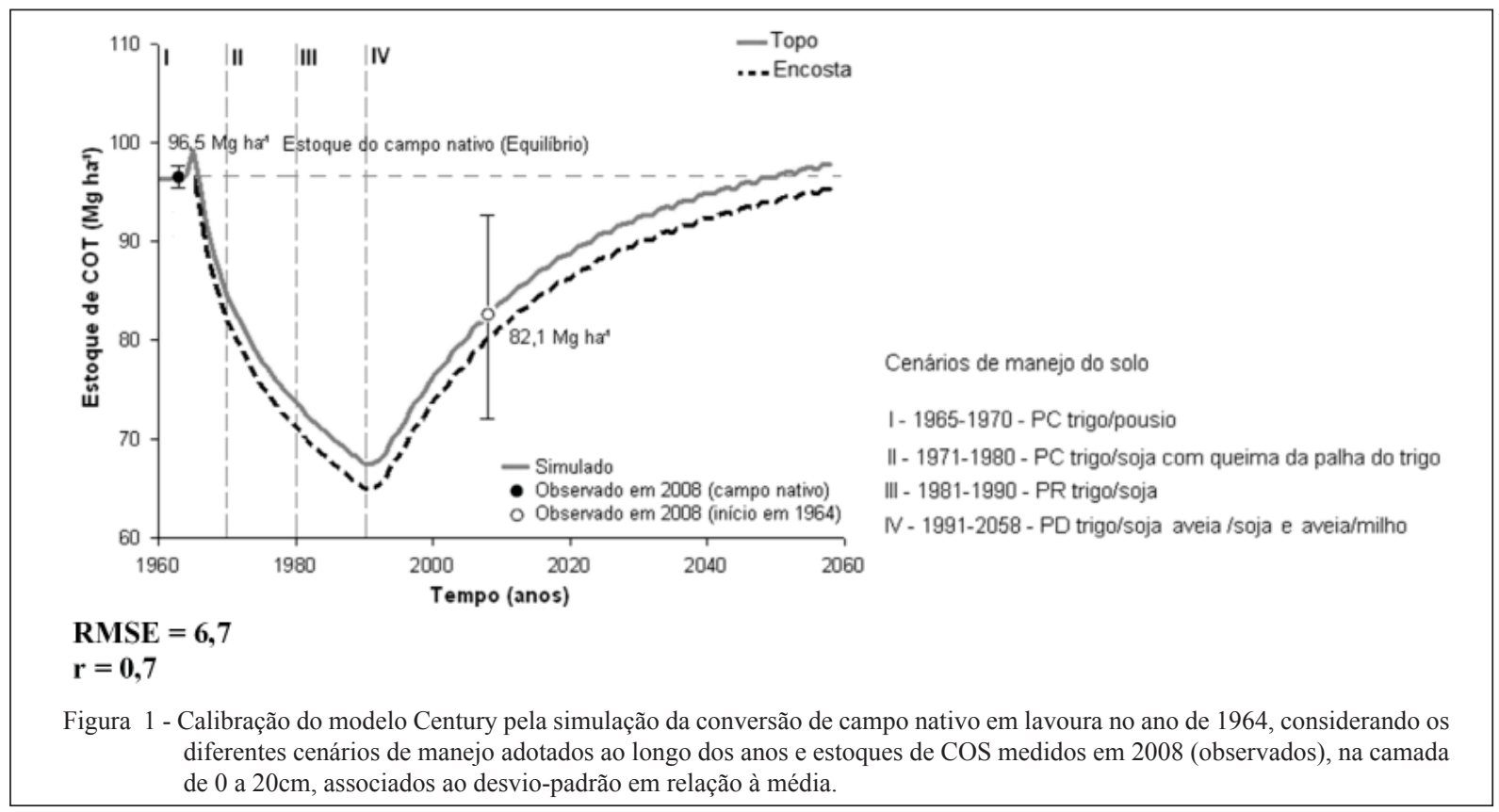

Ciência Rural, v.43, n.12, dez, 2013. 

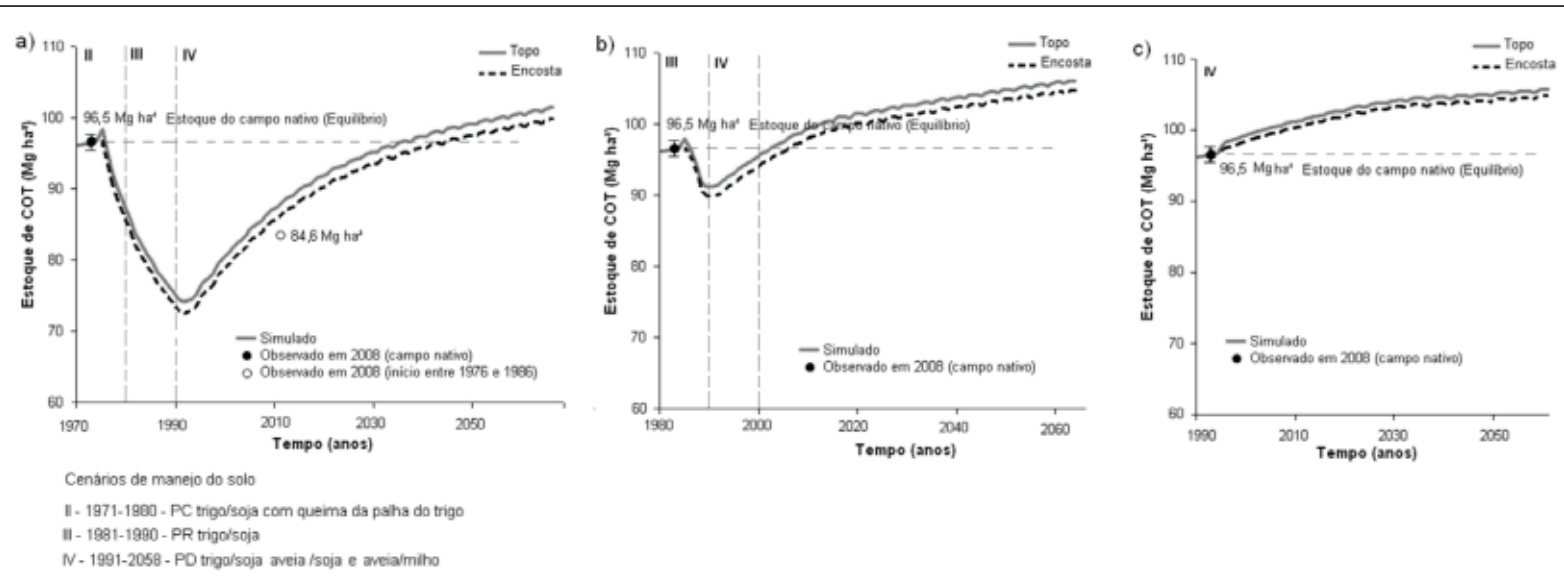

Figura 2 - Simulação dos estoques de COS para a unidade de simulação localizada em topo e encosta, com lavoura desde o ano de 1976 (a), 1986 (b) 1996 (c), em Vacaria, RS.

(2008), verificou-se um aumento de $15,2 \%$ no estoque de COS e, do período de 1992 até 2058, estimou-se um acréscimo de $26,9 \%$, os quais representam uma taxa de acúmulo de $0,41 \mathrm{Mg} \mathrm{ha}^{-1}$ ano $^{-1}$ e $0,78 \mathrm{Mg} \mathrm{ha}^{-1}$ ano ${ }^{-1}$ para os períodos de 1992 a 2008 e 1992 a 2058, respectivamente.

AUS 1987 (Figura 2b) apresentou as menores quedas, quando comparada com os estoques originais, devido ao menor tempo em que foram submetidas à exploração agrícola, sendo inexistente o período com a adoção de práticas não conservacionistas nessa unidade. As taxas de acúmulo de COS também não foram tão elevadas quando comparadas às taxas das outras US com lavouras mais antigas, e isso pode ter sido devido ao fato do menor período de exploração agrícola dessa unidade, quando comparado com as outras US, associado à adoção desde o início de sistemas conservacionistas de preparo e rotação/sucessão de culturas, não havendo assim grandes perdas de COS e, consequentemente, não sendo necessária uma grande recuperação dos estoques de COS. As taxas de acúmulo de COS estimadas para essa unidade de simulação foram de $0,44 \mathrm{Mg} \mathrm{ha}^{-1} \mathrm{ano}^{-1}$ entre os anos de 1992 a 2008, e de $0,22 \mathrm{Mg} \mathrm{ha}^{-1}$ ano $^{-1}$ para o período entre 1992 a 2058.

Nas lavouras iniciadas a partir de 1996 (Figura 2c), que foram implementadas diretamente sob PD, associado ao uso de culturas que proporcionam alto aporte de biomassa ao solo, não é verificada queda nos estoques de COS ao longo do tempo, ao contrário, verifica-se uma elevação nos estoques, quando comparados aos estoques da vegetação original. Essa condição já foi comprovada a campo por alguns autores (BAYER, 1996; DIEKOW et al., 2005), os quais verificaram que a adoção do $\mathrm{PD}$, juntamente com culturas com alta produção de biomassa, proporcionou um elevado aporte de $\mathrm{CO}$ ao solo, aumentando a qualidade do ambiente em regiões subtropicais. De acordo com SIX et al. (2002), os níveis de COS sob vegetação nativa podem não ser uma medida adequada para estimar a capacidade final de potencial armazenamento de $\mathrm{CO}$ nos solos, pois níveis nativos de COS podem refletir o equilíbrio de adição e perdas de $\mathrm{C}$, sob condições nativas, mas não necessariamente representam um limite superior no estoque de carbono no solo. As taxas de acúmulo de COS nesta unidade de simulação foram de $0,43 \mathrm{Mg}$ ha $^{-1}$ ano $^{-1}$ para os anos entre 1996 a 2008, e de $0,17 \mathrm{Mg}$ ha $^{-1}$ ano $^{-1}$ para o período entre 1996 a 2058.

Como pode ser visualizado na figura 2 , os estoques de COS estimados para o ano de 2008 ficaram acima do estoque original do campo nativo em todas as lavouras a partir do ano de 1987, quando os maiores estoques foram verificados nas lavouras iniciadas em 1996, apresentando valores 5,5\% a 4,8\% superiores aos estoques do campo nativo para topo e encosta, respectivamente.

A espacialização da distribuição do COS é uma aproximação da variabilidade dos valores reais de COS encontrados na região (Figura 3). À medida que for possível se introduzirem mais variáveis para compor as US, como, por exemplo, posições geomorfológicas do terreno e a distribuição espacial das áreas de erosão e deposição de sedimentos, melhor se poderá discretizar a variabilidade espacial do COS.

\section{CONCLUSÃO}

Verificou-se que o uso do modelo Century, previamente calibrado, associado a técnicas 


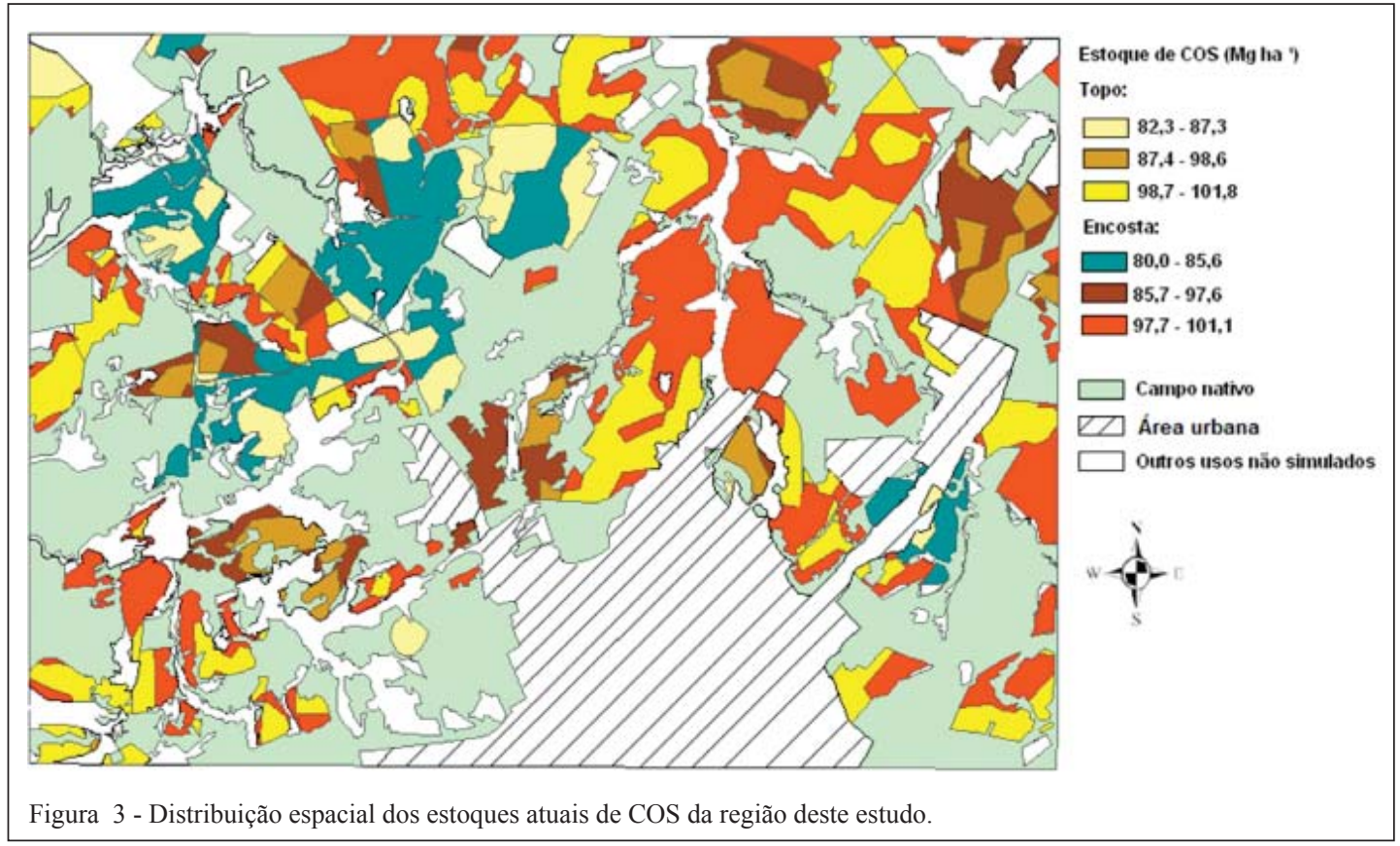

de sensoriamento remoto e geoprocessamento, possibilita estimar as alterações nos estoques originais de carbono causadas pelo uso agrícola do solo.

Sistemas conservacionistas de preparo do solo, associados à rotação de culturas com alto aporte de biomassa, mostraram-se armazenadores potenciais de estoques de CO nos solos da região de Vacaria, superiores aos estoques originais de campo nativo.

\section{REFERÊNCIAS}

BAYER, C. Dinâmica da matéria orgânica em sistemas de manejo de solos. 1996. 240f. Tese (Doutorado em Ciência do Solo) - Faculdade de Agronomia, Universidade Federal do Rio Grande do Sul, Porto Alegre, RS.

BORTOLON, E.S. O. et al. Simulação da dinâmica do carbono e nitrogênio em um argissolo do Rio Grande do Sul usando modelo century. R Bras Ci Solo, Viçosa, MG, 33, p.1635-1646, 2009. Disponível em: $\quad<$ http://www.scielo.br/scielo.php?script=sci arttext\&pid $=$ S0100-06832009000600012\&lng=pt\&nrm=iso $\&$ tlng=pt $>$. Acesso em: 16 ago 2010. doi: 10.1590/S010006832009000600012 .

BORTOLON, E.S. O. et al. Validation of the Century model to estimate the impact of agriculture on soil organic carbon in Southern Brazil. Geoderma, Amsterdam, v.167-168, p.156-166, 2011. Disponível em: <http://www.sciencedirect.com/science/ article/pii/S0016706111002497>. Acesso em: 10 ago 2012. doi: S0016706111002497.

BRASIL. Ministério da Agricultura. Departamento Nacional de Pesquisa Agropecuária. Divisão de Pesquisa Pedológica. Levantamento de reconhecimento dos solos do Estado do Rio Grande do Sul. Recife, 1973. 431p. (Boletim, 30).
DEBARBA, L. Simulação pelo Modelo Century do Impacto da Agricultura sobre o Estoque de Carbono Orgânico em Solos do Planalto Riograndense. 2002. 172 f. Tese (Doutorado em Ciência do Solo) - Programa de Pós-Graduação em Ciência do Solo, Faculdade de Agronomia, Universidade Federal do Rio Grande do Sul, Porto Alegre.

DIECKOW, J. et al. Soil $\mathrm{C}$ and $\mathrm{N}$ stocks as affected by cropping systems and nitrogen fertilization in a southern Brazil Acrisol managed under no-tillage for 17 years. Soil and Tillage Research, Amsterdam, v.81, p.87-95, 2005. Disponível em: <http://www. sciencedirect.com/science/article/pii/S0167198704001357>. Acesso em: 25 mar. 2009. doi: S0167198704001357.

EMBRAPA (EMPRESA BRASILEIRA DE PESQUISA AGROPECUÁRIA). Manual de métodos e análise de solo. 2.ed. Rio de Janeiro: CNPS, 1997. 212p.

LOPES, F. et al. Simulação da dinâmica do carbono do solo numa microbacia rural pelo modelo Century. Pesquisa Agropecuária Brasileira, Brasília, v.43, n.6, p.745-753, 2008. Disponível em: $\quad<$ http://www.scielo.br/scielo.php?pid=S0100$06832012000300014 \&$ script=sci_arttext $>$. Acesso em: 12 abr. 2009. doi: $10.1590 / \mathrm{S} 0100-06832012000300014$.

LOPES, F. et al. Evolução do uso do solo em uma área piloto da região de Vacaria; RS. Agriambi, Campina Grande, v.14, n.10, p.1038-1044, 2010. Disponível em: $<$ http://www.scielo.br/scielo. php?script=sci_pdf\&pid=S1415-43662010001000003\&lng=en\&n $\mathrm{rm}=$ iso\&tlng $=\mathrm{pt}>$. Acesso em: $10 \mathrm{dez} 2010$. doi: 10.1590/S141543662010001000003 .

PARTON, W.J et al. Analysis of factors controlling soil organic matter levels in great plains grasslands. Soil Science Society Of América, Madison, v.51, p.1173-1179, 1987. Disponível em: <https:// www.soils.org/publications/sssaj/pdfs/51/5/SS0510051173>. Acesso em: 25 mar. 2004. doi: 10.2136/sssaj1987.03615995005100050015x. 
POTTER, R.O. Caracterizacão de solos da região dos Campos de Cima da Serra - RS. 1977. 173f. Dissertacão (Mestrado em Ciência do Solo), Programa de Pós-graduação em Ciência do Solo, Faculdade de Agronomia, Universidade Federal do Rio Grande do Sul, Porto Alegre.

SIX, J. et al. Stabilization mechanisms of soil organic matter: Implications for C-saturation of soils. Plant and Soil. Amsterdam. v.241.p.155-176, 2002. Disponível em: <http://link.springer.com/ article/10.1023/A\%3A1016125726789>. Acesso em: 25 mar. 2004. doi: 10.1023/A\%3A1016125726789.
SMITH, P. et al. A comparation of the performance of nine soil organic matter models using datasets from seven long-term experiments. Geoderma, Amsterdam, v. 81, p.153-225, 1997. Disponível em: $<$ http:// www.sciencedirect.com/science/article/pii/S0016706197000876>. Acesso em: 25 mar. 2004. doi: S0016706197000876.

TORNQUIST, C.G. et al. Spatially explicit simulations of soil C dynamics in Southern Brazil: Integrating Century and GIS with i-Century. Geoderma, Amsterdam, v.150. p.404-414, 2009. Disponível em: $<$ http:// www.sciencedirect.com/science/article/pii/S0016706109000743>. Acesso em: 10 ago. 2010. doi: S0016706109000743.

Ciência Rural, v.43, n.12, dez, 2013. 